\title{
Periodontal health of London women during early pregnancy
}

\author{
S. Moore, ${ }^{1}$ M. Ide, ${ }^{2}$ R. F. Wilson, ${ }^{3}$ P. Y.Coward, ${ }^{4}$ E. Borkowska, ${ }^{5}$ R. Baylis, ${ }^{6}$ S. Bewley, ${ }^{7}$ \\ D. J. Maxwell, ${ }^{8}$ L. Mulhair, ${ }^{9}$ and F. P. Ashley ${ }^{10}$
}

Objectives A descriptive cross-sectional study to determine the severity of periodontal disease in early pregnancy and its relation to demographic variables in a South East London population.

Methods 2027 pregnant women attending Guy's Hospital for an ultrasound scan at 10 to 14 weeks gestation were assessed. Data were collected via questionnaire and periodontal examination, including plaque and bleeding scores, pocket probing depth and loss of attachment.

Results Mean age was 29.8 years (sd 5.5). Of these, $61.8 \%$ were white, $28.5 \%$ black, and $9.7 \%$ of other ethnic group. A total of $15 \%$ reported smoking during pregnancy. The mean number of teeth present was 28 (sd 2) per subject, mean percentage of sites with plaque present was $60.5 \%$ (sd 22.6), mean pocket depth was $2.0 \mathrm{~mm}$ (sd 0.4), mean loss of attachment was $0.4 \mathrm{~mm}$ ( $\mathrm{sd} 0.3$ ), and the mean percentage of sites bleeding on probing was $20.2 \%$ (sd 16.3). Linear regression demonstrated that probing depth was related to age, ethnicity, socioeconomic status and plaque score but not to smoking whereas loss of attachment demonstrated relationships with age, smoking status and plaque score but not ethnicity or socioeconomic status.

Conclusion There was a relatively high proportion of subjects with deep periodontal pockets in this pregnant population compared to the Adult Dental Health Survey 1998 but with similar levels of loss of attachment and percentage of sites with plaque present. Several demographic factors were associated with the level of periodontal disease in this population.

Pregnant women demonstrate an increased level of gingival inflammation compared to non-pregnant controls. ${ }^{1-7}$ There have also been studies that have shown an increased probing depth during pregnancy ${ }^{1,4}$ however others have failed to demonstrate this finding. ${ }^{3,5}$ A fairly consistent observation is that these changes occur in the absence of changes in oral hygiene indices. $^{2,3,4}$

There are several other risk factors that have been associated with periodontal disease. Periodontal disease is worse in older age groups, ${ }^{8}$ due to cumulative tissue destruction rather than an agerelated increased susceptibility to periodontal disease. Black ethnic groups have more severe periodontal disease than white but these differences often disappear if socioeconomic grouping is

$1,2,5,6,10^{*}$ Department of Periodontology and Preventive Dentistry, Floor 21 Guy's Tower, Guy's King's and St. Thomas' Dental Institute, King's College, London SE1 9RT; 3, ${ }^{4}$ Dental Clinical Research, Floor 17 Guy's Tower, Guy's King's and St. Thomas' Dental Institute, King's College, London SE1 9RT; ${ }^{8,}{ }^{9}$ Fetal Medicine Unit, Floor 15 Guy's Tower, Guy's and St. Thomas' Hospital Trust, London SE1 9RT

${ }^{*}$ Correspondence to: Mark Ide, Lecturer

email:mark.ide@kcl.ac.uk

REFEREED PAPER

Received 09.06.00; Accepted 30.07.01

(C) British Dental Journal 2001; 191: 570-573 taken into account. ${ }^{9}$ Socioeconomic group influences periodontal disease severity but again this association tends not to be as strong if oral hygiene and smoking are considered. ${ }^{9}$ Smoking remains a major risk factor for periodontal disease, ${ }^{8}$ even after adjusting for age, socioeconomic status and oral hygiene. However these factors do not explain all the potentially susceptible patients: the majority of periodontitis occurs in a minority of individuals. ${ }^{10}$

The aims of this study were to investigate the level of periodontal disease in women in early pregnancy and to identify associations with possible risk factors.

\section{Materials and Methods}

The study was approved by the Guy's Hospital local ethical committee. A total of 2027 pregnant women were assessed after attending for a routine nuchal translucency ultrasound scan as part of a larger study of periodontal disease in relation to pregnancy outcome. The majority of these women lived in London, from the Guy's and St Thomas' catchment areas, however a small number (approximately 5\%) were referred from Worthing Hospital, West Sussex as this centre does not perform nuchal translucency scans.

Of those that attended for this scan, $57.1 \%$ were screened. Exclusion criteria for recruitment included: subjects who required prophylactic antibiotics before dental treatment; multiple pregnancies; mothers who were less than 10 weeks or more than 15 weeks of gestation.

An examiner-administered questionnaire was completed for each subject including age, ethnicity and smoking status during pregnancy. Socioeconomic status was classified according to the Standard Occupational Classification of the Office of Population Censuses and Surveys (1990, 1991 and 1995) and recorded for both the mother and, if appropriate, her partner. Students were termed 'unclassified'.

The periodontal examination was performed by a trained examiner (SM) with the subject supine on a hospital bed. The light source was provided by a fibre optic light system (Universal Dual Quartz Halogen, Keeler Ltd, Windsor) with autoclavable light source tips (constructed at Scientific Workshops, Guy's \& St. Thomas' Hospital Trust) into which a disposable mirror head could be fitted (Mirodent, Guest Dental and Medical Products, Montefiori, Switzerland). A Hu-Friedy PQW manual periodontal probe (California, USA) with a tip diameter of $0.45 \mathrm{~mm}$ was used. Missing teeth and partially erupted teeth were recorded and the latter excluded from the periodontal assessment. For maxillary teeth mid-buccal and mesio-buccal sites were assessed, and for mandibular teeth mid-lingual and mesio-lingual sites were assessed. Clinical parameters measured at each site were: presence or absence of plaque (visible when removed by the probe), clinical pocket probing depth (in $\mathrm{mm}$ ), clinical loss of attachment from the base of the periodontal pocket to the cemento-enamel junction (in $\mathrm{mm}$ ), and bleeding on probing (presence or absence). 
An intra-examiner reproducibility study was performed. Fifty subjects, with a range of severity of periodontal disease were reexamined approximately 30 minutes after they were periodontally assessed for the study. The examiner was blind to the clinical data recorded at the first examination.

\section{Statistical Analysis}

Data were analysed using SPSS (Statistical Package for Social Sciences for Unix, SPSS Inc., Chicago, Illinois, USA) and Stata 7 for PC (Stata Corporation, College Station, Texas, USA).

Frequencies and distributions of variables studied were investigated. Those variables found not to be normally distributed were logarithmically transformed for parametric analysis.

The independent effects of age, ethnicity, socioeconomic group and smoking status on mean plaque score, mean bleeding score, mean probing depth, and mean loss of attachment were investigated. For this purpose, these variables were formed into binomial groups, with the exceptions of ethnicity and socioeconomic status. Subjects were divided by age into those less than and those equal to or greater than 30 years. Subjects were classified as belonging to either white, black or other ethnic groups. The socioeconomic status was taken as the highest of each subject and their partner, and divided into one of three groups, namely one group for those in socioeconomic classes I or II, one for classes III, IV, or V and a third which contained the unemployed and unclassifiable. Group $t$-tests were used to identify significant differences between the groups and analysis of variance was used similarly to investigate differences between ethnic and socioeconomic groups.

Linear regression analysis was performed to identify any of those independent variables which may predispose to increased periodontal disease allowing for interactions between such variables. The association with periodontal disease of age, ethnicity, socioeconomic status, number of cigarettes smoked and plaque score were investigated. The dependent variables of probing depth, loss of attachment, and bleeding on probing score were chosen as markers of periodontal disease status.

Intraclass correlation coefficients were determined for the reproducibility data.

\section{Results}

The results are based on the 2027 subjects recruited. Subjects were screened at a mean gestational age of 12.6 weeks. This was the first pregnancy for $43.8 \%$ of subjects. The mean age of the subjects was 29.8 (sd 5.5) years and ranged from 14 to 45 years.
Table I. Demographic data

\begin{tabular}{l|l|cc}
\hline & \multicolumn{3}{|c}{ n (total = 2027) } \\
\hline Ethnicity & White & 1252 & 61.8 \\
& Black Caribbean & 188 & 9.3 \\
& Black African & 294 & 14.5 \\
& Black Other & 96 & 4.7 \\
& Black Total & 578 & 28.5 \\
& Indian / Pakistani / Bangladeshi & 47 & 2.3 \\
& Chinese & 38 & 1.9 \\
& Asian - other & 18 & 0.9 \\
& Non-classified & 94 & 4.6 \\
& Other Total & 197 & 9.7 \\
\hline Socioeconomic group - & I/II & 1089 & 53.7 \\
highest between subject & III/IVN & 713 & 35.2 \\
and partner & Unemployed/unclassified & 225 & 11.1 \\
\hline & & & \\
Smoking during pregnancy & 304 & 15.0 \\
\hline
\end{tabular}

Table 2. Mean, standard deviation and range for the periodontal variables. $\mathbf{n}=\mathbf{2 0 2 7}$

\begin{tabular}{llll}
\hline & mean & sd & range \\
\hline Number of teeth & 27.9 & 2.4 & $10-32$ \\
Plaque score (\% of sites) & 60.5 & 22.6 & $1.8-100$ \\
Mean probing depth $(\mathrm{mm} / \mathrm{site})$ & 2.01 & 0.36 & $1.20-4.24$ \\
Mean loss of attachment $(\mathrm{mm} / \mathrm{site})$ & 0.38 & 0.32 & $0-2.82$ \\
Bleeding on probing (\% of sites) & 20.2 & 16.3 & $0-85.0$ \\
\hline
\end{tabular}

$17 \%$ (353) of the subjects were less than 25 years old, 20\% (398) were 35 years and over.

Table 1 shows the demographic data and Table 2 summarises the periodontal data. Table 3 compares mean periodontal measures by age, ethnicity, socioeconomic and smoking status groups. Subjects with higher mean plaque, bleeding scores and mean loss of attachment included those who were less than 30 years of age and those currently smoking. The smokers also had a higher mean probing depth. Analysis of variance indicated that there were significant differences between the three ethnic

Table 3. Mean (standard deviation) of periodontal variables in groups according to age, ethnicity, socioeconomic and smoking status (during pregnancy), with $p$ values from two-group $t$ tests. $n=2027$. $\S$ denotes $p$ value by ANOVA.

\begin{tabular}{|c|c|c|c|c|c|c|c|c|c|c|}
\hline Variable & & $\mathrm{n}$ & $\begin{array}{l}\text { Mean plaque } \\
\text { score } \\
\%(s d)\end{array}$ & $p$ value & $\begin{array}{l}\text { Mean bleeding } \\
\text { score } \\
\%(s d)\end{array}$ & $p$ value & $\begin{array}{l}\text { Mean probing } \\
\text { depth } \\
\mathrm{mm} / \text { site }(\mathrm{sd})\end{array}$ & $p$ value & $\begin{array}{l}\text { Mean loss of } \\
\text { attachment } \\
\mathrm{mm} / \mathrm{site}(\mathrm{sd})\end{array}$ & $p$ value \\
\hline \multirow[t]{2}{*}{ Age (years) } & $<30$ years & 898 & $63.4(21.8)$ & $<0.001$ & $22.5(16.7)$ & $<0.001$ & $2.00(0.33)$ & 0.245 & $0.32(0.27)$ & $<0.001$ \\
\hline & $\geq 30$ years & 1129 & $58.2(22.8)$ & & $18.4(15.8)$ & & $2.01(0.38)$ & & $0.42(0.35)$ & \\
\hline \multirow[t]{3}{*}{ Ethnicity } & White & 1252 & $60.0(22.7)$ & & $20.0(16.3)$ & & $1.95(0.34)$ & & $0.37(0.32)$ & \\
\hline & Black & 578 & $60.6(22.6)$ & $0.159 \S$ & $19.8(15.5)$ & $0.037 \S$ & $2.13(0.36)$ & $<0.001 \S$ & $0.39(0.32)$ & $0.222 \S$ \\
\hline & Other & 197 & $63.8(22.2)$ & & $23.1(18.5)$ & & $2.02(0.37)$ & & $0.40(0.35)$ & \\
\hline Socio- & $\mathrm{I} / \mathrm{II}$ & 1089 & $55.7(22.2)$ & & $17.6(15.2)$ & & $1.97(0.34)$ & & $0.37(0.31)$ & \\
\hline economic & III/IV/V & 713 & $65.7(21.8)$ & $<0.001 \S$ & $23.1(16.8)$ & $<0.001 \S$ & $2.04(0.36)$ & $<0.001 \S$ & $0.37(0.32)$ & $0.065 \S$ \\
\hline group & others & 225 & $67.6(21.6)$ & & $24.2(17.8)$ & & $2.09(0.40)$ & & $0.42(0.39)$ & \\
\hline \multirow[t]{2}{*}{ Smoking } & No & 1723 & $58.8(22.4)$ & $<0.001$ & $19.8(16.3)$ & 0.006 & $1.99(0.35)$ & $<0.001$ & $0.36(0.31)$ & $<0.001$ \\
\hline & Yes & 304 & $70.5(20.1)$ & & $22.6(16.4)$ & & $2.09(0.42)$ & & $0.47(0.38)$ & \\
\hline
\end{tabular}


groups for mean bleeding score and mean probing depth. Analysis of variance also revealed differences in mean plaque and bleeding score and mean probing depth between the three socioeconomic groups.

Table 4 summarises the results of the linear regression analysis. The final model demonstrated that probing depth was related to age, ethnicity, socioeconomic status and plaque score. The relationship of probing depth to smoking status approached significance $(\mathrm{p}=0.057)$. Loss of attachment showed a relationship with age, smoking status during pregnancy and plaque score. Bleeding score was related to age, socioeconomic status, smoking status, and plaque score.

The intraclass correlation coefficients for the intra-examiner reproducibility study were 0.90 for mean percentage of sites with visible plaque, 0.94 for percentage of sites bleeding on probing, 0.85 for mean probing depth and 0.98 for mean loss of attachment.

Table 4. Multiple linear regression models with $p$ values for the effect of independent variables with the adjusted $\mathbf{R}^{2}$ values for each model. $\mathbf{n}=\mathbf{2 0 2 7}$

\begin{tabular}{llll}
\hline & $\begin{array}{l}\text { Mean probing } \\
\text { depth }\end{array}$ & $\begin{array}{l}\text { Mean loss of } \\
\text { attachment }\end{array}$ & $\begin{array}{l}\text { Bleeding on } \\
\text { probing }\end{array}$ \\
\hline Age & $<0.001$ & $<0.001$ & 0.001 \\
Ethnicity & 0.001 & 0.132 & 0.579 \\
Socioeconomic group & 0.023 & 0.227 & 0.009 \\
Smoking & 0.057 & $<0.001$ & 0.021 \\
Plaque score & $<0.001$ & $<0.001$ & $<0.001$ \\
Adjusted R & 0.193 & 0.172 & 0.264 \\
\hline
\end{tabular}

\section{Discussion}

Previous studies on periodontal disease in pregnancy have tended to use different methods for measuring periodontal disease and loss of periodontal attachment was often not taken into account. Much of this work was performed 20-30 years ago, in other countries, and may not be representative of a current UK population. In the present study, only $17 \%$ of subjects were under 25 years. This is in contrast with the 1994-1995 NHS Maternity Statistics study where the equivalent figure was $27 \% .{ }^{11}$ Our population may differ from that in other areas of the country.

The periodontal variables in this study were compared with those for the Adult Dental Health Survey (ADHS) 1998. ${ }^{12}$ The ADHS study data was analysed by gender and age group therefore non-pregnant females aged 16 to 44 years could be compared to our pregnant females aged 14 to 45 years. It was found that $65 \%$ of our population had one or more sites with visible plaque and this figure is similar to that in the ADHS study of $71 \%$. Of this population of pregnant women $13.9 \%$ had one or more deep ( $6 \mathrm{~mm}$ or more) pockets compared to $2.9 \%$ of subjects in the ADHS study. Likewise there was a higher proportion of pregnant subjects with one or more probing depths of $4 \mathrm{~mm}$ or more $(75.8 \%$ compared to $48.3 \%) .{ }^{12}$ The higher proportion of subjects with deep periodontal pockets in the present study may be due to the influence of pregnancy hormones on development of false pocketing and consequently increased probing depths. ${ }^{3}$ However $1.8 \%$ of our population had one or more sites with loss of attachment of $6 \mathrm{~mm}$ or more compared to $1.9 \%$ in the ADHS study. ${ }^{12}$ Although both the present study and the Adult Dental Health Survey measured disease at buccal surfaces of maxillary teeth and the lingual surfaces of mandibular teeth, mesial and mid-tooth sites were assessed in the present study as opposed to mesial and distal sites in the ADHS. This may have resulted in an underestimate of the level of periodontal disease ${ }^{13}$ in our study. The results of the intra-examiner reproducibility study indicated a strong agreement in the measurement of the periodontal variables within study subjects.

Self-reported smoking in this study is lower than in other studies, ${ }^{14,15}$ possibly due to biased self-reporting in a hospital setting. Analysis demonstrated that those smoking during pregnancy had higher mean plaque scores, bleeding scores, mean probing depths and loss of attachment. However the relationship with mean probing depth was not statistically significant in the regression analysis $(p=0.057)$. Previous studies have generally found that oral hygiene is similar between smokers and nonsmokers, but that there is less bleeding in smokers. For example, Swenson ${ }^{16}$ and Bergström ${ }^{17}$ reported no significant differences in oral hygiene measures between the two groups, both before and after oral hygiene instruction, but a decreased percentage of bleeding points in smokers. In contrast, our results demonstrated poorer oral hygiene and increased mean bleeding score in smokers suggesting that the relationship between plaque, inflammation and smoking may be modified in pregnancy. The lack of a difference in probing depth between the current nonsmokers and smokers may be due to reduced clinical probing depth in smokers, which it has been suggested may be due to less inflammation and a difference in tissue consistency. ${ }^{18}$

The population in this study differs from that investigated in a previous study of periodontal disease and preterm low birth weight by Offenbacher et al (1996) in the USA. ${ }^{19}$ The study reported here featured subjects with a higher mean age, a lower proportion of Black ethnic groups and fewer smokers. Generally, there was a lower level of periodontal disease than in the USA study, however Offenbacher's group assessed all sites and the recorded level of periodontal disease may therefore be expected to be more severe than in the present study, which utilised partial recording. Even when allowances are made for this it would appear that Offenbacher's population was particularly prone to periodontal disease. These differences may affect the interpretation of relationships between periodontal disease and pregnancy outcome in the UK.

\section{Conclusion}

There was a higher proportion of subjects with deep periodontal pockets in this population than has been previously reported for general female UK populations. Linear regression techniques demonstrated that the level of periodontal disease, as assessed by probing depth, loss of attachment or by bleeding score was related to age, ethnicity, socioeconomic status, smoking in addition to plaque levels, and it is acknowledged that these factors can influence pregnancy outcome. It is therefore necessary to control for these variables when investigating associations between periodontal disease and preterm low birth weight.

This study was devised and carried out with the help and guidance of Professor Frank Ashley, who sadly passed away before the paper was accepted for publication. We wish to acknowledge the tremendous contribution that he made to setting up and running this project. The authors wish to acknowledge the assistance of colleagues and support staff in the Fetal Medicine Department and the Department of Periodontology and Preventive Dentistry for their help in this study and Colgate-Palmolive, UK for supplying oral hygiene packs for the subjects. This study is funded by a grant from the NHS Executive, London Region R \& D, grant number SPGS 593.

1. Löe H, Silness J. Periodontal disease in pregnancy, I. Prevalence and severity. Acta Odontol Scand 1963; 21: 533-551.

2. Silness J, Löe H. Periodontal disease in pregnancy, II. Correlation between oral hygiene and periodontal condition. Acta Odontol Scand 1964; 22: 121-135.

3. Cohen D W, Shapiro J, Friedman L, Kyle G C, Franklin S. A longitudina investigation of the periodontal changes during pregnancy and fifteen months post-partum: part II. J Periodontol 1971; 42: 653-657. 
4. Arafat A H. Periodontal status during pregnancy. J Periodontol 1974; 45: 641-643.

5. Samant A, Malik C P, Chabra S K, Devi P K. Gingivitis and periodontal disease in pregnancy. J Periodontol 1974; 47: 641-643.

6. Jago J D, Chapman P J, Aitken J F, McEniery T M. Dental status of pregnant women attending a Brisbane maternity hospital. Community Dent Oral Epidemiol 1984; 12: 398-401.

7. Miyazaki H, Yamashita Y, Goto-Kimura K, Shimada N, Sogame A, Takehara T. Periodontal condition of pregnant women assessed by CPITN. J Clin Periodontol 1991; 18: 751-754.

8. Ismail A I, Morrison E C, Burt B A, Caffesse R G, Kavanagh M T. Natural history of periodontal disease in adults: findings from the Tecumseh Periodontal Disease Study: 1959-1987. J Dent Res 1990; 69: 430-435.

9. Genco R J. Current view of risk factors for periodontal diseases. J Periodontol 1996; 67: 1041-1049.

10. Löe H, Anerud A, Boysen H, Morrison E. Natural history of periodontal disease in man. J Clin Periodontol 1986; 13: 431-440.

11. NHS Maternity Statistics: England 1989-1990 to 1994-1995. London: Department of Health, 1997.
12. Kelly M, Steele J, Nutall N, et al. Adult Dental Health Survey. Oral health in the United Kingdom, 1998. London: Office for National Statistics, 2000.

13. Agerholm D M, Ashley F P. Clinical assessment of periodontitis in young adults -evaluation of probing depth and partial recording methods. Community Dent Oral Epidemiol 1996; 24: 56-61.

14. Owen L, McNeill A, Callum C. Trends in smoking during pregnancy in England, 1992-7: quota sampling surveys. Br Med J 1998; 317: 728.

15. Foster K, Lader D, Cheesbrough S. Infant Feeding Survey 1995. London: HMSO, 1997.

16. Swenson H M. The effect of smoking on plaque formation. J Periodontol 1979; 50: 146-147.

17. Bergström J. Oral hygiene compliance and gingivitis expression in cigarette smokers. Scand J Dent Res 1990; 98: 497-503.

18. Biddle A J, Watts T L P, Palmer R M, Wilson R F. Comparison of periodontal probing validity in smokers and non-smokers. J Dent Res 1998; 77: 991 . Abstract 2874.

19. Offenbacher S, Katz V, Fertik G, et al. Periodontal infection as a possible risk factor for preterm low birth weight. J Periodontol 1996; 67: 11031113 . 